\title{
Wilson Prize article: Transverse phase-space manipulations in electron accelerators
}

\author{
Bruce E. Carlsten \\ Los Alamos National Laboratory, Los Alamos, New Mexico 87545, USA
}

(Received 22 July 2020; accepted 5 October 2020; published 13 November 2020)

\begin{abstract}
I am honored and grateful that my contributions to phase-space manipulations in general, and emittance compensation specifically, have been recognized by the Wilson Prize. The purpose of this article is to bring together what I believe to be the most important elements of the broad topic of transverse phase-space manipulations, focusing on the underlying beam physics which enable these types of schemes. We see that emittance compensation can be categorized as an example of the larger class of nonsymplectic manipulations. While the discussion is specifically focused on electron beams, much of the physics can also pertain to ion beams.
\end{abstract}

DOI: 10.1103/PhysRevAccelBeams.23.114801

\section{INTRODUCTION}

This article is largely intended to be a tutorial paper reviewing the key elements of transverse phase-space manipulations. While one main focus is providing a physical description of perhaps the most useful manipulation scheme, emittance compensation [1], it also includes a general discussion of the underlying beam physics of both symplectic and nonsymplectic phase-space manipulations. We will show that emittance compensation belongs to the class of nonsymplectic transformations.

In Sec. II, we provide the basic beam definitions and concepts needed for the following sections, including introducing the concept of the equivalent rms beam and that of a thermodynamic emittance. We describe the limitations to the thermodynamic model of emittance in Sec. III and we describe emittance compensation in Sec. IV. We point out the similarities and differences between symplectic-based manipulations and nonsymplectic-based manipulations (including emittance compensation) in Sec. V, along with a couple of examples.

\section{BASIC BEAM CONCEPTS}

A beam is a collection of particles mostly going in the same direction which we will call the longitudinal or axial

\footnotetext{
"The author received the American Physical Society's 2020 Robert R. Wilson Prize for Outstanding Achievement in the Physics of Particle Accelerators. This paper is an invited contribution inspired by that award.

bcarlsten@lanl.gov

Published by the American Physical Society under the terms of the Creative Commons Attribution 4.0 International license. Further distribution of this work must maintain attribution to the author(s) and the published article's title, journal citation, and DOI.
}

direction, as shown in Fig. 1. Understanding the transverse motion of particles is a major component of "beam physics" and can be very complex.

If the transverse motion is small enough relative to the longitudinal motion, we can use these canonical variables for each particle [2]:

$$
\vec{r}=\left(x, \frac{\gamma \beta_{x}}{\gamma_{0} \beta_{0}}, y, \frac{\gamma \beta_{y}}{\gamma_{0} \beta_{0}}, c \Delta t, \frac{\gamma-\gamma_{0}}{\gamma_{0} \beta_{0}}\right),
$$

where $\beta$ and $\gamma$ are the relativistic velocity and mass factor, " 0 " corresponds to some reference beam energy, and $\Delta t$ corresponds to the time a particle reaches some reference $z$ position. Using $x^{\prime}=d x / d z$ we rewrite the canonical variables in what we call phase (or trace) space variables:

$$
\vec{r}_{\text {phase space }}=\left(x, x^{\prime}, y, y^{\prime}, c \Delta t, \frac{\Delta(\gamma \beta)}{\gamma_{0}}\right) .
$$

We define rms beam parameters as the ensemble average of these quantities, such as

$$
\begin{aligned}
& x_{r m s}=\sqrt{\left\langle x^{2}\right\rangle} \\
& x_{r m s}^{\prime}=\sqrt{\left\langle x^{\prime 2}\right\rangle} .
\end{aligned}
$$

Important work by Sacherer [3] and Lapostolle [4] showed that the rms beam evolution mostly only depends on the beam's current rms quantities (which define the linear external and self-space-charge forces on the beam), to a very good approximation. This motivates a definition of the rms normalized emittance as a measure of beam quality (i.e., how long can a beam be kept how small), defined by

$$
\varepsilon_{x}=\gamma_{0} \beta_{0} \sqrt{\left\langle x^{2}\right\rangle\left\langle x^{\prime 2}\right\rangle-\left\langle x x^{\prime 2}\right\rangle}
$$




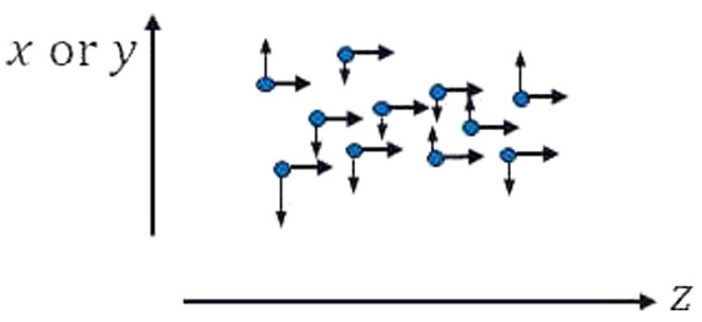

FIG. 1. For our purposes, a beam is a collection of particles that mostly go in the longitudinal, or $z$, direction, but with some transverse motion in the $x$ and $y$ directions.

in the $x$ direction (and an equivalent definition for the $y$ direction). (The $\gamma_{0} \beta_{0}$ terms add a normalization that makes the emittance definition independent of beam energy.) Consistent with convention in the high-brightness electron beam field, we will refer to the rms emittance simply as the emittance in the rest of this article.

Sacherer's and Lapostolle's work also allows us to think of the beam in terms of an equivalent uniform, hard-edged, rms beam with edge radius given by $r_{\text {edge }}=2 x_{r m s}$.

The emittance is related to the area of the beam's transverse-phase space, or

$$
\begin{aligned}
A_{\text {phase-space }} & \approx 4 \pi \frac{\varepsilon_{x}}{\gamma_{0} \beta_{0}} \\
& \approx 4 \pi \sqrt{\left\langle x^{2}\right\rangle\left\langle x^{\prime 2}\right\rangle-\left\langle x x^{\prime 2}\right\rangle},
\end{aligned}
$$

where the phase-space area of the equivalent rms beam is shown in Fig. 2.

Liouville's theorem [5] states that phase-space density is invariant, which naively might imply that the emittance in Eq. (4) is invariant. However, the emittance represents the phase-space area of the equivalent rms beam and not that of the actual beam distribution. While the local phase-space

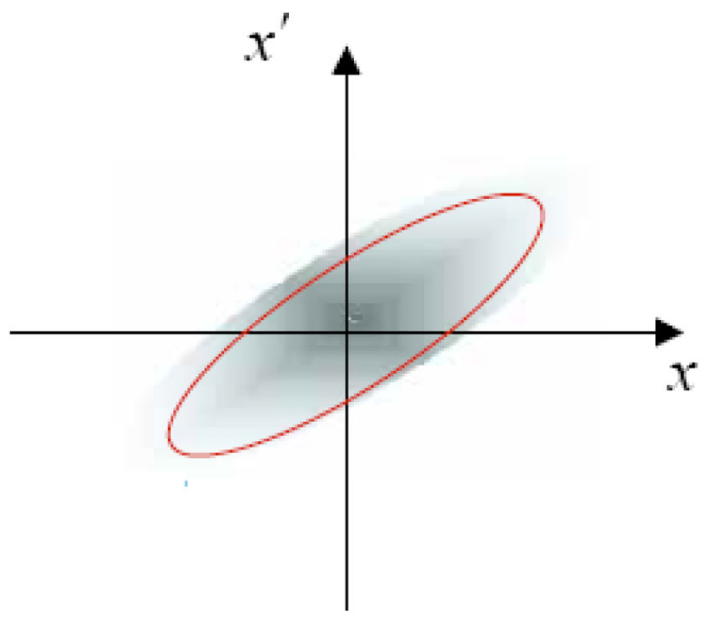

FIG. 2. Comparison of a phase-space distribution of particles (dots) and the equivalent hard-edged rms beam (uniform density within the red ellipse). This figure is courtesy of William Barletta. density near an individual electron is invariant under Liouville's theorem, the distribution of these densities can evolve. Despite this, the rms emittance is largely conserved along an accelerator since the beam mostly experiences linear forces. Motivated by this, beam physicists have often considered a transverse beam temperature that is equivalent to the beam emittance [6]. A transparent definition of this temperature is provided in [7],

$$
T_{\text {transverse }}=\varepsilon_{x}^{2} \frac{m c^{2}}{k_{B} \gamma_{0} x_{r m s}^{2}},
$$

where $m$ is the electronic mass, $c$ is the speed of light, and $k_{B}$ is Boltzmann's constant.

Using an equivalent temperature implies the emittance is thermodynamic in nature. This concept has been reinforced by the concept of "nonlinear free energy" [6,8], where a change in the beam's excess energy $U$ (defined by the sum of the beam's actual kinetic energy, space-charge potential energy, and the external potential energy less the sum of the energies for a beam with the same rms quantities but in the lowest energy configuration, i.e., a "stationary state") is related to the change in beam emittance by

$$
\frac{d \varepsilon_{x}^{2}}{d z}=-4 x_{r m s}^{2} \frac{2 I / I_{A}}{\beta \gamma^{3}} \frac{d}{d z}\left(\frac{U}{W}\right)
$$

where $I_{A}$ is about $17 \mathrm{kA}$ and $W$ is a constant equal to the beam current squared divided by $16 \pi \epsilon_{0} \beta^{2} c^{2}$ and where $\epsilon_{0}$ is the permittivity of free space. This theoretical expression was confirmed by Kehne's PhD thesis work [9] (Fig. 3), where five individual beamlets were merged and the resulting emittance growth was shown to be due to the decrease in the beam's nonlinear free energy.

Motivated by the nonlinear free-energy concept, the emittance is often thought as an entropic thermodynamic quantity, where increasing beam disorder driven by the available nonlinear free energy always leads to an emittance increase. The time constant for this exchange from nonlinear free energy to emittance growth is typically considered to be a quarter betatron period [6].

\section{LIMITS OF THE THERMODYNAMIC INTERPRETATION OF EMITTANCE}

The purpose of phase-space manipulations is to reduce the beam emittance. Such a reduction violates an entropic thermodynamic basis for the emittance. In this section, we introduce three common physical processes electrons can experience that enable rms emittance reductions.

\section{A. "Unwinding" the radial beam distribution before wave breaking}

As pointed out earlier, Liouville's theorem states that the local phase-space density stays constant, not that the rms 

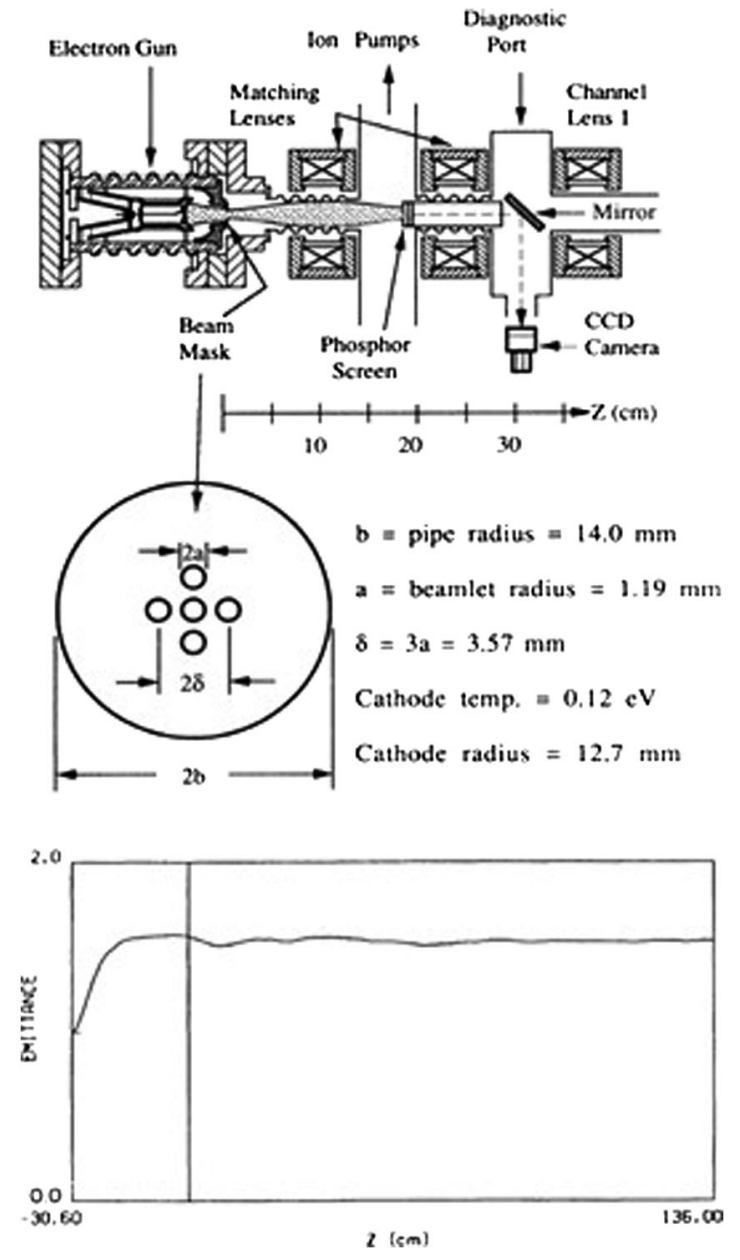

FIG. 3. Kehne's PhD thesis experiment. (Top) experimental setup; (middle) description of the five beamlets; (bottom) relative emittance growth due to nonlinear free energy. (Images from [9].)

emittance is invariant. In Fig. 4, we see the radial phase space of an electron beam in an induction linac at three places along the beam line [10]. There is some initial nonlinearity just after the electron gun (top left) due to nonlinear space charge arising from the nonuniform emission across the cathode surface and suppression of the axial electric field due to the anode aperture. Radially nonlinear focusing forces from a beam-capture solenoid also add to this initial nonlinear phase-space distribution. Note that the beam is essentially uniform over its entire axial length and all axial effects can be ignored for a long electron beam in an induction linac. This allows us to separate out radial effects from other effects.

In Fig. 4, we see three different levels of nonlinearities. First, just after the electron gun (top left), the distribution is slightly nonuniform, and even though the rms emittance is much larger than the distribution's actual area in phase space (which is close to zero), the distribution is largely single valued. Some initial wrapping of the distribution in phase space can be seen in the top-right image, where now
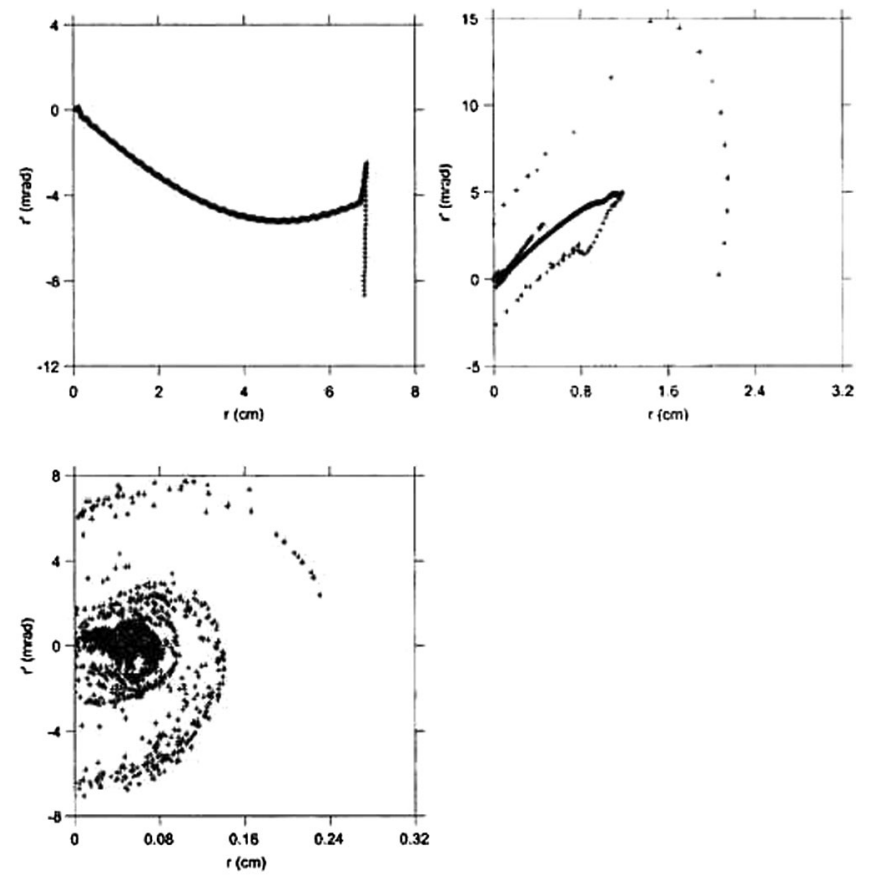

FIG. 4. Numerically modeled radial phase space of a beam in an induction linac [10]. (Top left) just after the electron gun, about $4 \mathrm{MeV}$; (top right) after a few $\mathrm{MeV}$ of acceleration and betatron periods; (bottom) at the end of the linac, about $20 \mathrm{MeV}$; images are from [10].

the distribution is starting to become multivalued in $r^{\prime}$ at most radial positions. The distribution is tightly wound in the bottom image. We can call the process of becoming multivalued "wave breaking" in the sense that some particles start overtaking others in configuration space. Importantly, while the beam's emittance had even grown a significant relative amount evolving to the distribution in the top-left plot (assuming the original emittance was zero as the beam's electrons' initial radial divergences all started at zero at the surface of the cathode), it is possible to reverse that emittance growth as long as the beam distribution stays single valued and has not started to wave break. We can also see why the length scale for conversion from nonlinear free energy to emittance is a quarter betatron period as that is the distance where particles in the beam's more tenuous outside region would be focused past particles that started at smaller initial radii, crossing their trajectories and leading to a multivalued distribution. Any nonzero beam emittance leads to some amount of wave breaking in a focusing system. Considering the balance between linear external focusing and the beam's repulsive space charge, a large fraction of the beam would wave break if the beam rms emittance exceeds a wave-breaking threshold given roughly by

$$
\varepsilon_{\mathrm{WB}-\mathrm{thres}} \sim 8 x_{r m s} \sqrt{\frac{I / I_{A}}{\gamma_{0} \beta_{0}}}
$$


which is typically reached in ion accelerators but not in electron accelerators with rf photoinjectors. Wave breaking in the context of rf photoinjectors is discussed in great detail in Anderson's excellent analysis of it [11].

\section{B. Variations along a bunch's axial direction}

Here, we consider a rotation of a very narrow axial slice of phase space as we move axially along a bunch of electrons as in Fig. 5, where $\zeta$ is the axial coordinate in the beam frame. Even though the emittance as defined by Eq. (4) can vanish if we only include electrons at or very near a given $\zeta$ value, the emittance over the entire bunch can become very large (for this example, the phase space at a given $\zeta$ value is a line with zero area but the phase space of the entire bunch fills a circle). Here we can differentiate between a "slice" emittance (where the emittance is calculated for a small axial slice of the bunch) and a "projected" emittance, where we are integrating over all bunch axial positions $\zeta$.

An important emittance growth of this nature occurs in a drifting slug of beam [12], where the radial space-charge force of the center of the beam is larger than that at the ends, either because of the short axial nature of the bunch or from an actual current variation along the bunch. The radial expansion of the beam as a function of $\zeta$ due to space charge is then a function of axial position $\zeta$,

$$
\ddot{r}_{\text {slice }}(\zeta)=\frac{F_{\text {Space charge }}(\zeta)}{\gamma m}=2 \frac{I(\zeta) / I_{A}}{r_{\text {slice }}(\zeta) \beta \gamma^{3}} c^{2},
$$

where we are explicitly showing the current, space-charge force, and slice radius all as functions of the axial position within the bunch, and where the dots indicate a time derivative. A bunch with an initially uniform radius then will expand out more in its center as it drifts, as in Fig. 6.

Kim extended the analysis approach introduced in [12] to estimate the space-charge induced emittance growth in an rf photoinjector [13]. Importantly, wave breaking is suppressed for this type of beam expansion and subsequent focusing as the electrons in different slices will not axially overtake each other (as all electrons become relativistic

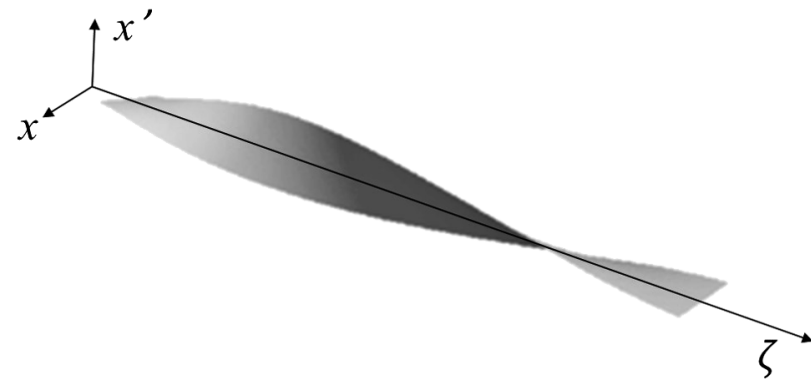

FIG. 5. Illustration of how the horizontal phase space can be twisted along the bunch's axial direction.
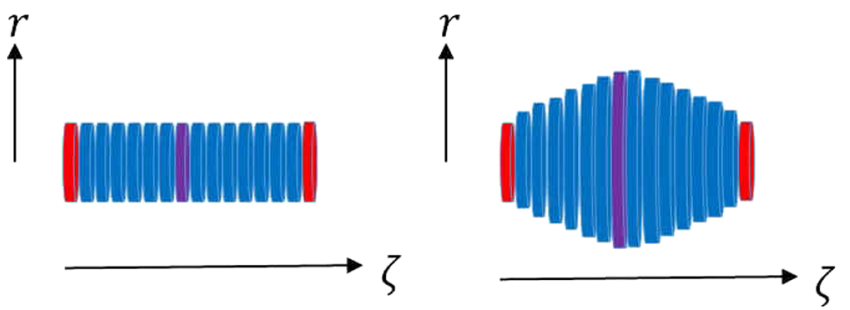

FIG. 6. (Left) bunch initially with uniform radius; (right) the center slice (purple) will expand more than the end slices (red) due to a higher space-charge force.

quickly), and the slice beam emittance is typically much lower than the threshold for wave breaking [Eq. (8)]. As a result, this emittance growth is reversible using emittance compensation, discussed in Sec. IV.

\section{Nonsymplectic interactions}

The third phase-space dynamic we include that cannot be explained by a thermodynamic basis for the beam emittance is if a nonsymplectic interaction is introduced into the beam. The earliest such technique was initially proposed by Bovet in 1970 [14] and described in detail by Peterson [15]. Here, a foil tapered horizontally is placed in the beam line. Particles passing through the foil lose energy, and since the foil is thicker on one horizontal end than the other, a correlation is generated between horizontal position $x$ and energy as shown in Fig. 7, taken from [15]. A dispersive section is then used to change the particles' horizontal position as a function of their energy. If adjusted correctly, the bunch can be compressed horizontally and since the horizontal divergence can be made independent of this action, the horizontal emittance is reduced. Section V has a more complete discussion of symplectic and nonsymplectic phase space manipulations. We shall see in the following

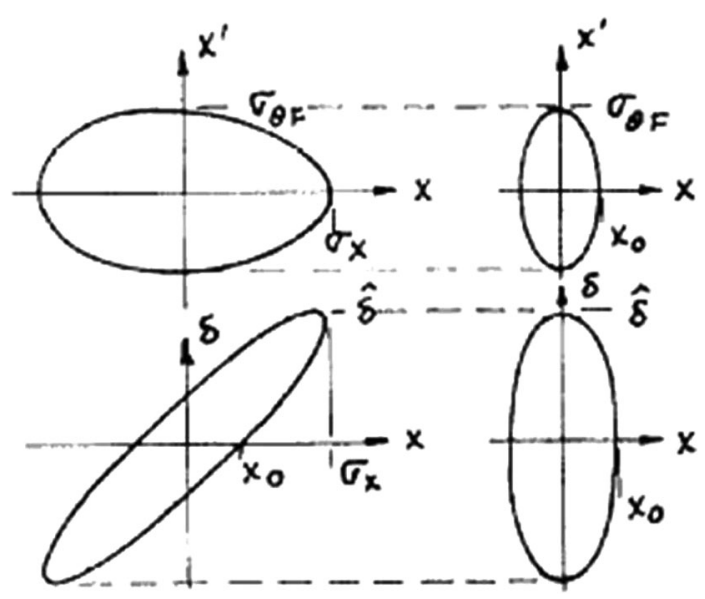

FIG. 7. One of Peterson's figures from [15] showing the initial (just after the tapered foil) and final horizontal phase spaces (top) and the initial and final $x$-energy correlations (bottom). 
sections that emittance compensation is in fact another example of a nonsymplectic interaction.

\section{EMITTANCE COMPENSATION}

Emittance compensation $[1,16]$ refers to the unwinding of a bunch's phase space for a short slug of beam (often with a current variation) along its axial direction such that the twisted strip shown in Fig. 5 becomes flat again. For the first part of this discussion, we assume the beam expansion due to space charge is limited by focusing from a uniform axial magnetic field to help illustrate the beam physics. We consider slices along the slug of beam as in Fig. 6 and, in Fig. 8, we see the orbits of the end slice edges (in red) and the center slice edge (in purple) in radial phase space. Here, the external magnetic field is adjusted so the end slices are initially focused but the center slice expands a bit. Thus, the red circle represents the rotation of the end slices about their equilibrium radius in phase space and the purple circle represents the rotation of the center slice about its equilibrium radius. The black lines scribe out the entire bunch's projected phase space, leading to a triangular shape representing a relatively large projected emittance growth. It is worth noting that while the projected bunch emittance cannot be thought of in thermodynamic terms, the emittance of each slice can be (with an exception described in the last paragraph of this section).

Of course, the same emittance growth mechanism occurs in the absence of an external magnetic field as suggested by Fig. 6, with now the black triangle in Fig. 8 lying at an angle between the $r$ and $r^{\prime}$ axes. In [1], it was shown that an emittance growth arising from the expansion of the bunch

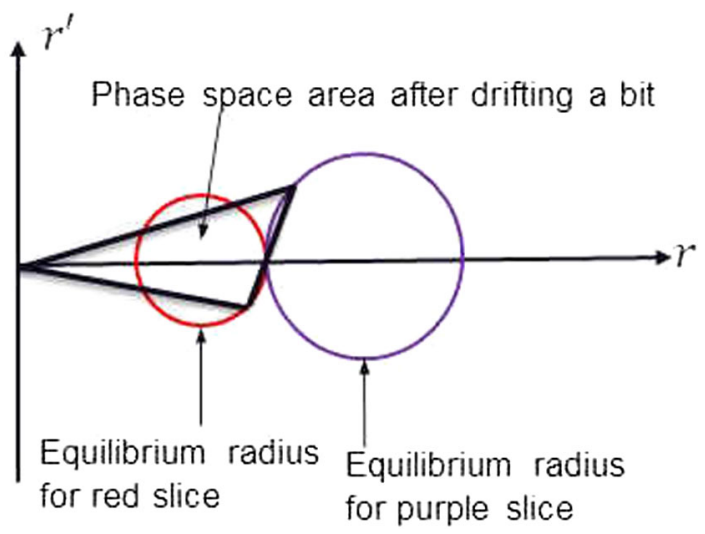

FIG. 8. Starting at the same radius (where the red and purple circles touch), the edge of the end slice will oscillate about a smaller radius (red circle) than the edge of the middle slice (purple circle). The electron orbits are clockwise in radial phase space, and the boundary of the bunch's projected phase space is represented by the black triangle after the bunch drifts a while. As the colored circles represent the radii of the edges of the center and edge slices, other particles from those slices lie on the two mostly horizontal black lines. slices in that case could be compensated by an appropriate downstream focusing element. In particular, a lens with focal length

$$
\frac{1}{f}=2 \frac{z_{1}+z_{2}}{z_{2}^{2}}
$$

will cause the space-charge forces after the lens to counter the twisting of the phase space caused by the space-charge forces before the lens, where $z_{1}$ is the drift distance to the lens from a position of initially minimum projected emittance and $z_{2}$ is the distance from the lens to the emittance minimum caused by emittance compensation. This theory was developed to design the first low-emittance rf photoinjector which set new high-brightness records $[17,18]$. The emittance reduction for a drifting slug beam was verified by detailed particle-in-cell simulations done by a team at SLAC [19], shown in Fig. 9.

Careful slice-resolved measurements showing the relative rotations of slice rms phase-space ellipses within an electron bunch were taken at an $\mathrm{rf}$ photoinjector at Brookhaven National Laboratory which verified the emittance compensation theory application to rf photoinjectors [20], shown in Fig. 10. Importantly, these measurements identified two emittance minimums occurring either for different lens focusing strengths at a single axial position or at different axial positions for the same focusing strength.

Serafini and Rosenzweig [21] provided an important advance in the understanding of emittance compensation by noting that the rotation period of the orbits in phase space depends on the external focusing, but not the current of a slice, which is easy to show for the special case of axially uniform focusing and no acceleration (as in Fig. 8). Specifically, if we consider the slice edge radial equation of motion,

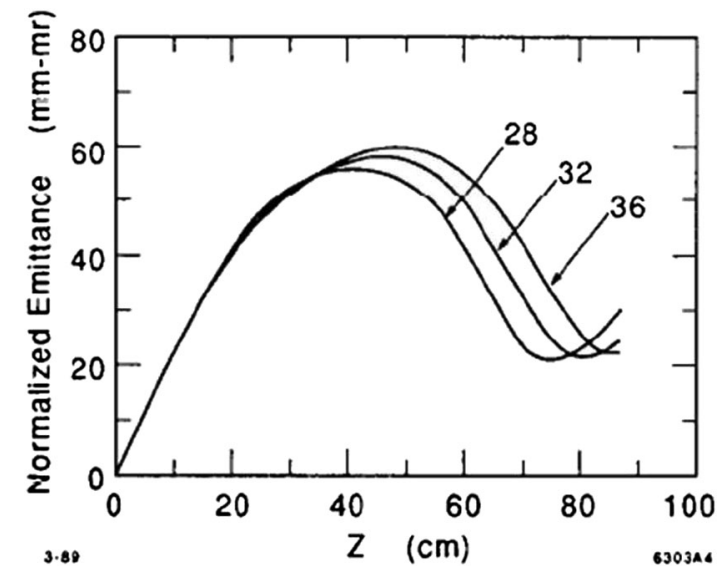

FIG. 9. SLAC simulations (from [19]) verifying the ability to compensate the emittance growth from axially dependent spacecharge forces. The numbers in the plot indicate the location of the lens in $\mathrm{cm}$. 

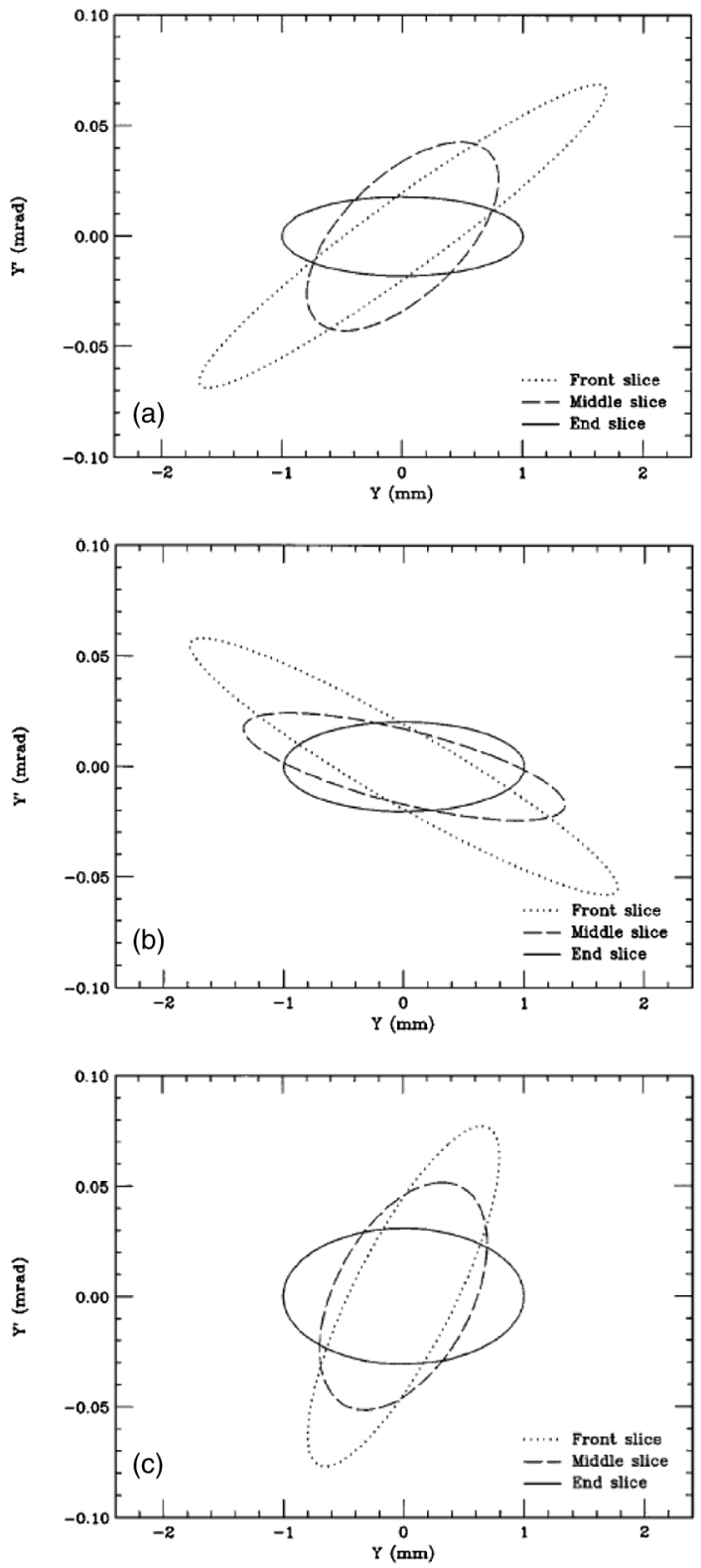

FIG. 10. Experimental BNL measurements (from [20]) showing the slice phase-space ellipses of the front, middle, and end slices, for solenoid currents of $102 \mathrm{~A}$ (a), $106 \mathrm{~A}$ (b), and $110 \mathrm{~A}$ (c). As predicted, the slices rotate relative to each other as the solenoid focal length is changed. The projected emittance is minimized at a solenoid current of $106 \mathrm{~A}$.

$$
r_{\text {slice }}^{\prime \prime}+K r_{\text {slice }}-\frac{K_{\text {space-charge }}(\zeta)}{r_{\text {slice }}}=0,
$$

where the primes indicate axial derivatives as before, $K$ represents a uniform, linear external focusing, $K_{\text {space-charge }}$ is the normalized space-charge force, and expand the radius about an equilibrium radius

$$
r_{\text {slice }}=r_{\text {slice,equil }}+\delta \text {, }
$$

where the slice equilibrium radius is a function of both the slice current and the external focusing

$$
r_{\text {slice,equil }}=\sqrt{K_{\text {space-charge }}(\zeta) / K}
$$

we find the period of the slice edge oscillation about its equilibrium radius is independent of the slice's current from the solution of

$$
\delta^{\prime \prime}+2 K \delta=0 .
$$

This is an important result and shows the slice oscillations all stay in phase and that the phase-space boundary as indicated in Fig. 8 will collapse to zero area every half oscillation period and form emittance minimums.

The focusing in a real rf photoinjector is a cross between a discrete lens and axially uniform focusing, but the main features discussed above are still valid. Simulations show there are two emittance minimums, and the conventional approach now is to place a booster accelerator at the location of the peak between the first two emittance minimums (known as the "Ferrario working point") and to "freeze" the emittance at the level of the second minimum as the beam becomes relativistic [22,23]. An example of an optimized rf photoinjector design from [11] is shown in Fig. 11 using the Ferrario working point.

Interestingly, it turns out that emittance compensation can also reduce emittance growth within individual slices due to radial nonlinearities of the type shown in Fig. 4 (top left) [10], as long as the variation is not too large to cause wave breaking. It is easy to see the same fundamental process occurs radially if, in Eqs. (11)-(14), we let the parameters $r, K, K_{\text {space-charge, }}$ and $\delta$ all be radial functions of their position within the slice.

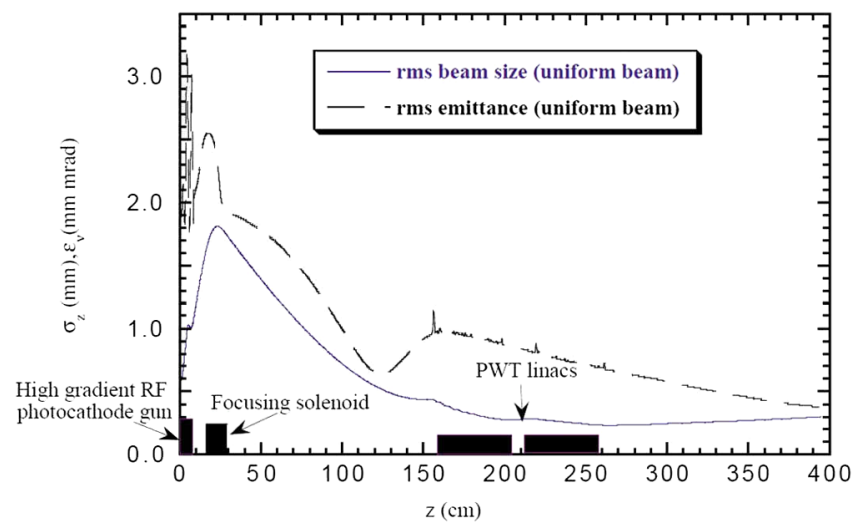

FIG. 11. Optimized rf photoinjector design using the Ferrario working point, from [11]. 


\section{EIGEN-EMITTANCES, SYMPLECTIC, AND NONSYMPLECTIC INTERACTIONS}

Now we will look at some properties of projected emittances. Importantly, a beam's eigen-emittances [24] are conserved under symplectic transformations [2,24,25] (which essentially includes all beam transport with the exception of space-charge forces and higher order beam line elements). The eigen-emittance concept is important because once all $x-y$ correlations are removed, the transverse beam emittances are equal to the eigen-emittances. Thus, the eigen-emittances concept gives us a tool to describe the effect of phase-space manipulations.

\section{A. Symplectic manipulations}

A flat-beam transformer (FBT) [26-30] is an example of applying the concept of eigen-emittances for manipulating beam emittances. In an FBT, a nonzero axial magnetic field at the beam cathode can be used to decrease one transverse emittance at the expense of the other, while keeping the product constant. The configuration of an FBT is shown in Fig. 12. In an FBT, the magnetic field on the cathode adds a canonical angular momentum to the beam, leading initially to transverse emittances of

$$
\varepsilon_{\text {trans }}=\sqrt{\mathbf{L}^{2}+\varepsilon_{0}^{2}}
$$

where $\mathbf{L}$ is due to the angular momentum [31],

$$
\mathbf{L}=\frac{e\left|B_{\text {cath }}\right|}{8 \gamma \beta c m} R_{\text {cath }}^{2}
$$

and $\varepsilon_{0}$ is the intrinsic emittance of the gun (i.e., what the transverse emittances would be in the absence of the axial magnetic field on the cathode). Three skew quadrupoles are used to remove all $x-y$ correlations (such that $\langle x y\rangle,\left\langle x y^{\prime}\right\rangle$, $\left\langle x^{\prime} y\right\rangle$, and $\left\langle x^{\prime} y^{\prime}\right\rangle$ all vanish) and the final $x$ and $y$ emittances become these eigen-emittances (for the case the angular momentum part is much larger than the intrinsic emittance)

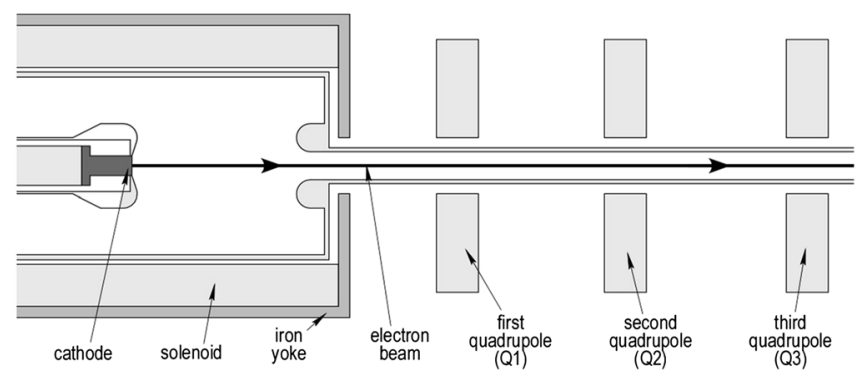

FIG. 12. Flat-beam transform configuration. A solenoid generates an axial magnetic field at the location of the cathode. Once the beam leaves the solenoidal field, it encounters three skew quadrupole which remove the $x-y$ correlations. This figure is courtesy of Kip Bishofberger.

$$
\begin{aligned}
\varepsilon_{\text {eig, }-} & =\frac{\varepsilon_{0}^{2}}{2 \mathbf{L}} \\
\varepsilon_{\text {eig },+} & =2 \mathbf{L} .
\end{aligned}
$$

Note that now one transverse emittance can be much larger than the other while keeping the product constant. To date, FBTs have only been demonstrated on rf photoinjectors, with a typical final emittance ratio $\varepsilon_{\text {eig, }+} / \varepsilon_{\text {eig,-- }}$ of about 100. Reference [30] reports for the first time the development of an FBT for a low-voltage, high-frequency, sheet-beam traveling-wave tube, where the emittance in the beam's narrow dimension must be reduced for achieving stable transport and for ensuring decent interaction between the electron beam and the rf structure $[32,33]$. Because the eigen-emittances are recovered at low energy, the beam's space-charge forces change the skew quadrupole strengths for recovering the eigen-emittances and additionally lead to growth in the eigen-emittances.

Figure 13 shows a simulation of the LCLS photoinjector [34,35] operating as an FBT, from [36]. Here, an axial magnetic field of $140 \mathrm{G}$ was imposed on the cathode, leading to an angular momentum contribution of $\mathbf{L}=$ $0.62 \mu \mathrm{m}$ with an intrinsic emittance of $\varepsilon_{0}=0.72 \mu \mathrm{m}$, for a bunch charge of $500 \mathrm{pC}$.

The eigen-emittances are recovered at $135 \mathrm{MeV}$ (past the second LCLS linac section) and are 0.203 and $1.64 \mu \mathrm{m}$. While we only see a rather modest emittance bump near the cathode in Fig. 13 compared to a typical rf photoinjector simulation (e.g., Fig. 11), the emittance compensation still occurs though it is conceptually harder to understand the dynamics with a magnetized beam. An important observation of this study was that the product of the final emittances (and thus of the intrinsic emittances) was somewhat lower than the product of the emittances that would occur if the cathode magnetic field vanished (in other words, the emittance growth of the effective intrinsic emittances was reduced). This is likely because the

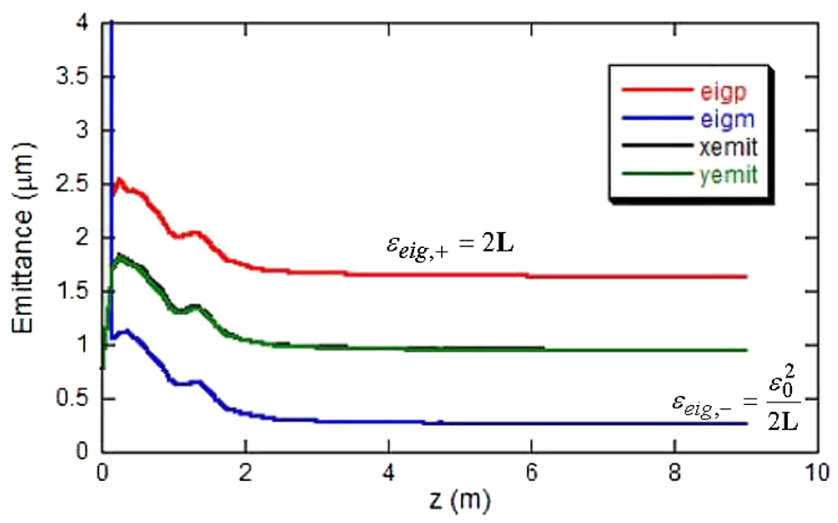

FIG. 13. Emittance evolution in the LCLS injector operated as an FBT where eigp is the higher ("plus") eigen-emittance, eigm is the lower ("minus") eigen-emittance, and the $x$ and $y$ projected emittances lie on top of each other (from [36]). 
magnetic field near the cathode suppressed beam expansion, space-charge emittance growth, and the resulting nonlinear forces from the rf fields.

A second symplectic manipulator is an emittance exchanger (EEX) [37,38], where two doglegs and a transversely deflecting cavity exchange the emittance between one transverse direction and the longitudinal direction. Emma et al. [39] has proposed using a combination of an FBT and an EEX to move excess emittance from both transverse directions into the longitudinal direction for future light sources. The steps would be: (i) Start with a super-short pancake of charge, with emittances of 2.1/2.1/0.15 $\mu \mathrm{m}$ (for $\varepsilon_{x} / \varepsilon_{y} / \varepsilon_{z}$ ), all in a magnetized rf gun (assuming a total volume of about $0.7 \mu \mathrm{m}^{3}$, which is about right for $1 \mathrm{nC}$ of bunch charge). (ii) Use an FBT to adjust these numbers to $0.15 / 30 / 0.15 \mu \mathrm{m}$. (iii) Use an EEX to swap the $y$ and $z$ emittances and end up with 0.15 / $0.15 / 30 \mu \mathrm{m}$ for the final $\varepsilon_{x} / \varepsilon_{y} / \varepsilon_{z}$.

Complications with this approach are that the phase space volume (the $0.7 \mu \mathrm{m}^{3}$ ) is not truly independent of the actual initial partitioning and also some applications need low $z$ emittances. However, this approach may be appropriate for some applications and certainly demonstrates the power of symplectic phase-space manipulations. (It is worth noting that this approach is superior to simply starting with a long beam with a very small radius to reduce the initial transverse emittances due to time varying rf effects.)

\section{B. Nonsymplectic manipulations}

The Lorentz force law follows from the single-particle Hamiltonian

$$
H=c \sqrt{[\vec{p}-q \vec{A}(\vec{r}, t)]^{2}+m^{2} c^{2}}+q \phi(\vec{r}, t)
$$

which in turn depends on the position and velocities of all the $N$ particles within the beam (through the vector and scalar potentials, which in general also include external forces). If the $6 \mathrm{~N}$ Hamiltonian reduces to a quadratic Hamiltonian in beam coordinates (i.e, the beam transfer matrix $R$ is linear), then we can write the rms symplectic condition

$$
J_{6}=R^{T} J_{6} R,
$$

where $J_{6}$ is the six-dimensional unit symplectic matrix [31], and the eigen-emittances do not change. However, if the Hamiltonian is higher order in particle coordinates, the rms symplectic condition [Eq. (19)] no longer follows and the eigen-emittances are no longer preserved (as, for example, in Bovet's scheme and, interestingly enough, also in the emittance compensation process since each slice sees a different space-charge force). In [36], the LCLS injector FBT was followed by a modern version of Bovet's approach (Fig. 14), where a canted undulator provided a

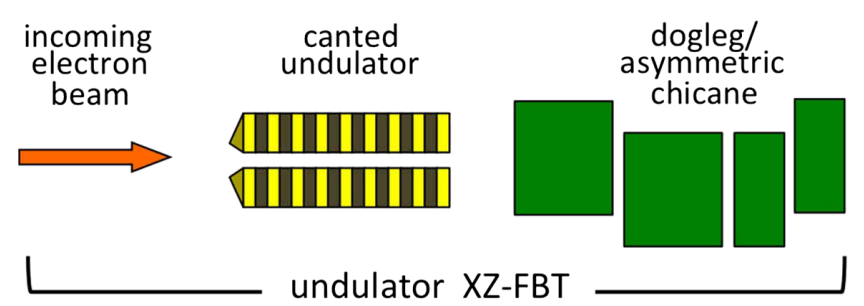

FIG. 14. More modern concept of the Bovet/Peterson emittance reduction technique. Reference [36] used this configuration to develop a scheme to transfer excess transverse emittance into the longitudinal direction both to reduce the transverse emittances and to provide a substitute for the laser heater for the LCLS accelerator. The image is from [42].

correlation between a particle's horizontal position and energy. Incoherent synchrotron radiation was used as the nonsymplectic transform, as the undulator field was stronger at one horizontal side of the canted undulator than the other. This transformation is nonsymplectic because there is a change in energy linear with horizontal position but there is not a change in horizontal divergence linear with axial position. A more sophisticated beam optics section was used to recover the eigen-emittances than what Peterson had, based on our more mature understanding of eigen-emittances. We can recognize this section (either a dogleg or an asymmetric chicane) as a version of an FBT that removes $x-z$ correlations. In Bovet's/ Peterson's configuration, the amount of emittance reduction was limited by the energy straggling from the interaction of the electrons with the foil. Here, an equivalent limitation arises from the energy spread induced by the quantum fluctuations of the incoherent synchrotron radiation. For this configuration, final transverse emittances for the central $250 \mathrm{pC}$ of the bunch of $\varepsilon_{x} / \varepsilon_{y}=$ $0.27 / 0.19 \mu \mathrm{m}$ were achieved, which correspond to about a factor of 6 reduction in the transverse emittance product over the standard LCLS rf photoinjector and linac operation at $250 \mathrm{pC}$. The longitudinal energy spread increased by about a factor of 20 (and the overall phase space volume was increased by a factor of about 3 ). In practice, this increased energy spread does not cause a problem since the energy spread of the standard LCLS beam is "heated" with a laser anyway to suppress the microbunch instability [40,41]. In fact, this scheme could be used to simultaneously reduce the transverse emittances of the beam while replacing the laser heater.

\section{CONCLUSIONS}

The understanding of emittance manipulations and phase-space partitioning has matured greatly over the past three decades. For manipulating projected emittances, single-stage, multistage, symplectic, and nonsymplectic schemes are all well understood with an understanding based on eigen-emittances. 
Since each bunch slice sees a different space-charge force, emittance compensation is in the larger class of nonsymplectic transformations. It is significantly different than the other nonsymplectic examples presented since the interaction occurs over a long distance instead of being limited to a single location. Using the beam's space-charge forces to eliminate the axially dependent rotation angles in phase space that they previously caused works surprisingly well. To date, emittance compensation is unique in being the only phase-space manipulation scheme currently in wide use. Emittance compensation has helped rf photoinjectors achieve their full potential and has been a critical beam line element in achieving the successful operation of most X-ray free-electron lasers in the world.

New niche applications for phase-space manipulations are emerging including developing highly asymmetric emittance beams for high-frequency vacuum electronics [30] which we described earlier. I think it is likely that these types of schemes will become as common as emittance compensation in the future.

\section{ACKNOWLEDGMENTS}

The author would like to acknowledge the truly paradigm-shifting contribution by Richard Sheffield and John Fraser in inventing the rf photoinjector [43]. The discussion of phase-space manipulations only makes sense in the context of the extraordinarily bright beams produced by these devices.

[1] B. E. Carlsten, New photoelectric injector design for the Los Alamos National Laboratory XUV FEL accelerator, Nucl. Instrum. Methods Phys. Res., Sect. A 285, 313 (1989).

[2] B. E. Carlsten, K. A. Bishofberger, L. D. Duffy, S. J. Russell, R. D. Ryne, N. A. Yampolsky, and A. J. Dragt, Arbitrary emittance partitioning between any two dimensions for electron beams, Phys. Rev. ST Accel. Beams 14, 050706 (2011).

[3] F. J. Sacherer, RMS envelope equations with space charge, IEEE Trans. Nucl. Sci. 18, 1105 (1971).

[4] P. Lapostolle, A. Lombardi, and T. P. Wangler, A model of nonlinear space charge forces in a charged particle beam, Report No. CERN-PS-91-11-HI, 1993.

[5] J. D. Lawson, The Physics of Charged-Particle Beams (Oxford University Press, New York, 1977).

[6] M. Reiser, Theory and Design of Charged Particle Beams (Wiley, New York, 1994).

[7] M. Zuboraj and B. Carlsten, Beam transport and beamcurrent loss in emittance-dominated high-frequency tubes, IEEE Trans. Electron Devices 66, 3599 (2019).

[8] T. P. Wangler, K. R. Crandall, R. S. Mills, and M. Reiser, Relation between field energy and rms emittance in intense particle beams, in Proceedings of the 11th Particle Accelerator Conference, PAC-1985, Vancouver, BC, Canada, 1985 (IEEE, New York, 1985).
[9] I. Haber, D. Kehne, M. Reiser, and H. Rudd, Experimental, theoretical, and numerical investigation of the homogenization of density nonuniformities in the periodic transport of a space-charge dominated beam, Phys. Rev. A 44, 5194 (1991).

[10] B. E. Carlsten, Long-term, correlated emittance decrease in intense, high-brightness induction linacs, Phys. Plasmas 6 , 3615 (1999).

[11] S. G. Anderson and J. B. Rosenzweig, Nonequilibrium transverse motion and emittance growth in ultrarelativistic space-charge dominated beams, Phys. Rev. ST Accel. Beams 3, 094201 (2000).

[12] M. E. Jones and B.E. Carlsten, Space-charge induced emittance growth in the transport of high-brightness electron beams, in Proceedings of the 1987 Particle Accelerator Conference (IEEE, Washington, DC, 1987).

[13] K. J. Kim, Rf and space-charge effects in laser-driven rf electron guns, Nucl. Instrum. Methods Phys. Res., Sect. A 275, 201 (1989).

[14] C. Bovet, Increasing the energy spread of the beam by traversal of a thin foil, LBL Internal Report No. ERAN 89, 1970.

[15] J. M. Peterson, Reduction of beam emittance by a tapered-foil technique, IEEE Trans. Nucl. Sci. 30, 2403 (1983).

[16] B. E. Carlsten, Space-charge induced emittance compensation in high-brightness photoinjectors, Part. Accel. 49, 27 (1995).

[17] B. E. Carlsten, L. M. Young, M. E. Jones, B. Blind, E. M. Svaton, K. C. D. Chan, and L. E. Thode, Accelerator design and calculated performance of the Los Alamos HIBAF facility, Nucl. Instrum. Methods Phys. Res., Sect. A 296, 687 (1990).

[18] D. W. Feldman, S. C. Bender, B. E. Carlsten, J. Early, R. B. Feldman, W. J. D. Johnson, A. H. Lumpkin, P. G. O'Shea, W. E. Stein, R. L. Sheffield, and L. M. Young, Experimental results from the Los Alamos FEL photoinjector, IEEE J. Quantum Electron. 27, 2636 (1991).

[19] H. Hanerfeld, W. Herrmannsfeldt, and R. Miller, Higher order correlations in computed particle distributions, in Proceedings of the 1989 Particle Accelerator Conference, Chicago, IL (IEEE, New York, 1989).

[20] X. Qiu, K. Batchelor, I. Ben-Zvi, and X.-J. Wang, Demonstration of Emittance Compensation through the Measurement of the Slice Emittance of a 10-ps Electron Bunch, Phys. Rev. Lett. 76, 3723 (1996).

[21] L. Serafini and J. B. Rosenzweig, Envelope analysis of intense relativistic quasilaminar beams in rf photoinjectors: A theory of emittance compensation, Phys. Rev. E 55, 7565 (1997).

[22] M. Ferrario, J. E. Clendenin, D. T. Palmer, J. B. Rosenzweig, and L. Serafini, HOMDYN study for the LCLS RF photoinjector, Report No. SLAC-PUB-8400, 2000.

[23] C. X. Wang, J. K. Kim, M. Ferrario, and A. Wang, Criteria for emittance compensation in high-brightness photoinjectors, Phys. Rev. Accel. Beams 10, 104201 (2007).

[24] A. J. Dragt, F. Neri, and G. Rangarajan, General moment invariants for linear Hamiltonian systems, Phys. Rev. A 45, 2572 (1992). 
[25] L. D. Duffy and A. J. Dragt, Utilizing the eigen-emittance concept for bright electron beams, Adv. Imaging Electron Phys. 193, 1 (2016).

[26] Y. Derbenev, Adapting optics for high energy electron cooling, University of Michigan Report No. UM-HE-9804, 1998.

[27] R. Brinkmann, Ya. Derbenev, and K. Floettmann, A low emittance, flat-beam electron source for linear colliders, Phys. Rev. ST Accel. Beams 4, 053501 (2001).

[28] P. Piot, Y.-E. Sun, and K.-J. Kim, Photoinjector generation of a flat electron beam with transverse emittance ratio of 100, Phys. Rev. ST Accel. Beams 9, 031001 (2006).

[29] B. E. Carlsten and K. A. Bishofberger, Simple algorithm for designing skew quadrupole emittance converter configurations, New J. Phys. 8, 286 (2006).

[30] S. Moroch, B. Carlsten, and T. Koeth, Space-charge effects in low-energy flat-beam transforms (to be published).

[31] K.-J. Kim, Round-to-flat transformation of angularmomentum-dominated beams, Phys. Rev. ST Accel. Beams 6, 104002 (2003).

[32] B. E. Carlsten, K. E. Nichols, D. Y. Shchegolkov, and E. I. Simakov, Emittance effects on gain in W-band TWTs, IEEE Trans. Electron Devices 63, 4493 (2016).

[33] B. E. Carlsten, T. Koeth, F. L. Krawczyk, S. Moroch, K. Nichols, E. Simakov, and M. R. Zuboraj, High-bandwidth, high-power Ka-band TWT design with a ceramic slowwave structure (to be published).

[34] P. Emma, First lasing and operation of an Ångstrom-wavelength free-electron laser, Nat. Photonics 4, 641 (2010).

[35] C. Limborg-Deprey and P. Emma, Alternative tunings for the linac coherent light source photoinjector, in Proceedings of the 21st Particle Accelerator Conference, Knoxville, TN, 2005 (IEEE, Piscataway, NJ, 2005).
[36] B. E. Carlsten, K. A. Bishofberger, L. D. Duffy, J. W. Lewellen, Q. R. Marksteiner, and N. A. Yampolsky, Using emittance partitioning instead of a laser heater to suppress the microbunch instability, IEEE Trans. Nucl. Sci. 63, 921 (2016).

[37] M. Cornacchia and P. Emma, Transverse to longitudinal emittance exchange, Phys. Rev. ST Accel. Beams 5, 084001 (2002).

[38] T. W. Koeth et al., Emittance exchange at the Fermilab A0 photoinjector, in Proceedings of the 11th European Particle Accelerator Conference, Genoa, 2008 (EPS-AG, Genoa, Italy, 2008).

[39] P. Emma, Z. Huang, K.-J. Kim, and P. Piot, Transverse-tolongitudinal emittance exchange to improve performance of high-gain free-electron lasers, Phys. Rev. ST Accel. Beams 9, 100702 (2006).

[40] Z. Huang et al., Measurements of the linac coherent light source laser heater and its impact on the x-ray free-electron laser performance, Phys. Rev. ST Accel. Beams 13, 020703 (2010).

[41] M. Borland, Start-to-end simulation of self-amplified spontaneous emission free electron lasers from the gun through the undulator, Nucl. Instrum. Methods Phys. Res., Sect. A 483, 268 (2002).

[42] K. Bishofberger, Emittance-partitioning strategies for future accelerator applications, at the Proceedings, 26th International Linear Accelerator Conference (LINAC12): Tel Aviv, Israel, September 9-14, 2012, edited by M. Draper, C. Carli, J. Poole, L. Liljeby, and S. Lupu (JaCOW, Geneva, Switzerland, 2012).

[43] J. S. Fraser, R. L. Sheffield, E. R. Gray, and G. W. Rodenz, High-brightness photoemitter injector for electron accelerators, IEEE Trans. Nucl. Sci. 32, 1791 (1985). 